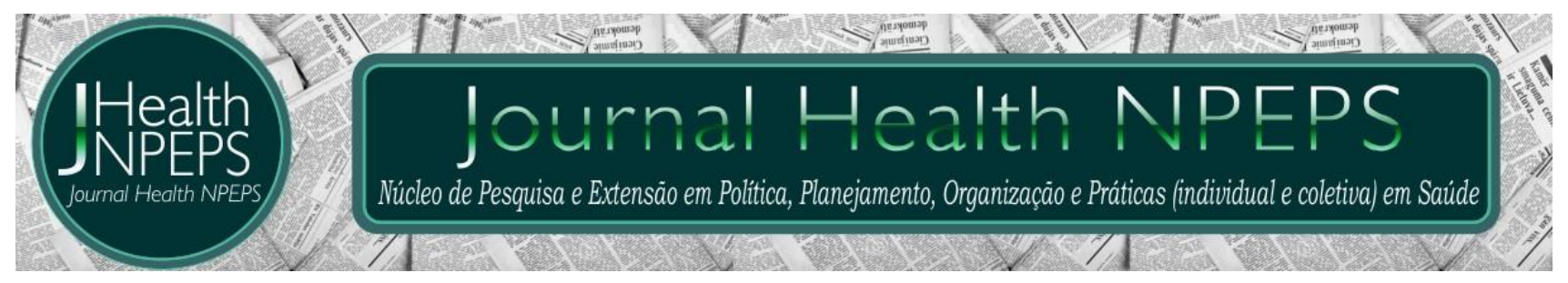

http://dx.doi.org/10.30681/252610105320

ARTIGO ORIGINAL

\title{
Relação dos biomas nos acidentes peçonhentos no Brasil
}

\author{
Relationship of biomes in venomous accidents in Brazil
}

Relación de biomas en accidentes venenosos en Brasil

\section{Francisco Cezar Aquino de Moraes ${ }^{1}$, Alison Ramos da Silva ${ }^{2}$, Emanuele Rocha da Silva ${ }^{3}$, Johne de Souza Coelho ${ }^{4}$, Pedro Pereira de Oliveira Pardal ${ }^{5}$}

\begin{abstract}
RESUMO
Objetivo: descrever o perfil dos casos de acidentes por animais peçonhentos no Brasil, de 2010 a 2019, para verificar a relação dos biomas brasileiros sobre o número de notificações. Método: estudo exploratório, a partir de dados secundários do Departamento de Informática e Informação do Sistema Único de Saúde quanto ao número de casos absolutos de acidentes por Estados e Distrito Federal, distribuídos em mapa coroplético. Pela correlação linear de Pearson estimou a relação entre número de casos e desmatamento. Resultados: os principais acidentes notificados envolveram escorpiões $(52,14 \%)$, principalmente no cerrado e na caatinga, seguido por aranhas $(16,23 \%)$ majoritariamente no pampa, e serpentes $(15,45 \%)$ na amazônia. Esses últimos com letalidade superior a 1\%. Os Estados que apresentaram o maior número de casos novos foram Minas Gerais (49.716), São Paulo (41.428), Bahia (24.617) e Pernambuco (21.144). As variáveis de desmatamento foram inversamente proporcionais ao número de casos, principalmente nos Estados com maior notificação. Conclusão: o grande registro de acidentes por escorpiões e a alta letalidade associada aos acidentes por serpente nos biomas brasileiros representam potencial problema de saúde pública. No entanto, não há indícios de correlação positiva entre desmatamento e número de notificações dos acidentes.
\end{abstract}

Descritores: Animais Venenosos; Ecossistema; Análise Espacial.

\section{ABSTRACT}

\footnotetext{
${ }^{1}$ Acadêmico de Medicina. Universidade Federal do Pará (UFPA). Belém, PA, Brasil. E-mail: francisco.aquino.moraes@ics.ufpa.br ORCID ID: https://orcid.org/0000-0003-0623-8135

2Biólogo. Mestre em Biologia Parasitária na Amazônia. Universidade Federal do Pará (UFPA). Belém, Pará, Brasil. E-mail: alisonramos23@gmail.com ORCID ID: https://orcid.org/0000-0002-1080-5545

${ }^{3}$ Acadêmica de Medicina. Universidade Federal do Pará (UFPA). Belém, PA, Brasil. E-mail: emanuele.silva@ics.ufpa.br ORCID ID: https://orcid.org/0000-0002-9109-4158

${ }^{4}$ Médico. Mestre em Doenças Tropicais. Universidade Federal do Pará (UFPA). Belém, Pará, Brasil. E-mail: johnecoelho@gmail.com ORCID ID: https://orcid.org/0000-0002-6929-4807

${ }^{5}$ Médico. Doutor em Medicina Tropical. Coordenador do Laboratório de Entomologia Médica e Animais Peçonhentos do Núcleo de Medicina Tropical, Universidade Federal do Pará (UFPA). Belém, Pará, Brasil. E-mail: pepardal@ufpa.br ORCID ID: https://orcid.org/0000-0002-4405-281X Autor para correspondência - Endereço: Avenida Generalíssimo Deodoro, 92, 66055-240, Belém, PA, Brasil.
} 
Objective: to describe the profile of cases of accidents by venomous animals in Brazil, from 2010 to 2019, to verify the relationship of Brazilian biomes on the number of notifications. Method: exploratory study, using secondary data from the Department of Informatics and Information of the Unified Health System regarding the number of absolute cases of accidents by States and the Federal District, distributed in a choropleth map. Pearson's linear correlation was used to estimate the relationship between the number of cases and deforestation. Results: the main accidents reported involved scorpions (52.14\%), mainly in the cerrado and caatinga, followed by spiders (16.23\%) mainly in the pampa, and snakes (15.45\%) in the amazon. These with a lethality higher than 1\%. The states with the highest number of new cases were Minas Gerais (49,716), São Paulo (41,428), Bahia $(24,617)$ and Pernambuco $(21,144)$. Deforestation variables were inversely proportional to the number of cases, especially in the states with the highest reporting. Conclusion: the great record of accidents by scorpions and the high lethality associated with snake accidents in Brazilian biomes represent a potential public health problem. However, there is no evidence of a positive correlation between deforestation and the number of accident reports.

Descriptors: Poisonous Animals; Ecosystem; Spatial Analysis.

\section{RESUMEN}

Objetivo: describir el perfil de casos de accidentes por animales venenosos en Brasil, de 2010 a 2019, para verificar la relación de biomas en el número de notificaciones. Método: estudio exploratorio utilizando datos del Departamento de Informática e Información del Sistema Único de Salud sobre el número de casos absolutos de accidentes por estados y Distrito Federal, distribuidos en mapa de coropletas. La correlación lineal de Pearson estimó la relación entre el número de casos y la deforestación. Resultados: los principales accidentes reportados fueron de escorpiones (52,14\%), principalmente en el cerrado y caatinga, seguidos de arañas $(16,23 \%)$, principalmente en pampa y serpientes $(15,45 \%)$, la mayoría en la amazonía. Los estados con el mayor número acumulado de nuevos casos fueron Minas Gerais (49.716), São Paulo (41.428), Bahía (24.617) y Pernambuco (21.144). Las variables de deforestación por número de casos fueron inversamente proporcionales, especialmente en los estados con mayor reporte. Conclusión: la gran cantidad de accidentes de escorpiones y la alta letalidad de los accidentes de serpientes en los biomas brasileños representan un problema potencial de salud pública. Sin embargo, no hay evidencia de una correlación positiva entre la deforestación y el número de informes de accidentes.

Descriptores: Animales Venenosos; Ecosistema; Análisis Espacial.

\section{INTRODUÇÃO}

Animais peçonhentos são vertebrados ou invertebrados capazes de produzir veneno (peçonha) e de injetá-lo em presas através de dentes diferenciados - aguilhões, quelíceras, ferrões e outras estruturas -, sendo que, em humanos, seu veneno pode causar dolorosas intoxicações, e até mesmo levar a óbito caso não haja socorro imediato da vítima. Os animais peçonhentos que mais causam acidentes no território brasileiro são escorpiões, serpentes, aranhas, lagartas e abelhas, e constituem um particular problema de saúde pública nos países tropicais e subtropicais, pela 
subnotificação/incompletude de registro de casos e pela gravidade de sintomas que podem causar nos pacientes ${ }^{1,2}$.

No território brasileiro, desde o início do processo de colonização, há modificação do meio ambiente pelo homem, principalmente sob influência da demanda decorrente dos ciclos econômicos, sendo a continuada expansão dos centros urbanos no sentido do litoral para o continente influenciador nesse processo de modificação ambiental e desmatamento ${ }^{3}$.

0 número de acidentes por animais peçonhentos vem aumentando ao longo dos anos nas grandes cidades e nas regiões menos urbanizadas, não estando mais necessariamente confinados ao meio rural. Assim sendo, a rápida transformação no espaço geográfico levou à sobreposição do homem e destes animais no ambiente, podendo levar os últimos à adaptação à vida urbana ${ }^{4}$. Nesse escopo, a notificação de acidentes por animais peçonhentos está distribuída amplamente pelos biomas brasileiros, que segundo o Instituto Brasileiro de Geografia e Estatística (IBGE) correspondem à amazônia, caatinga, cerrado, mata atlântica, pampa e pantanal ${ }^{5}$.
O conhecimento epidemiológico acerca dos acidentes envolvendo animais peçonhentos no território brasileiro é de grande importância, em especial para os profissionais da área da saúde, que poucas vezes recebem informações dessa natureza no decorrer da formação acadêmica ou de atuação profissional ${ }^{6}$.

Nesse sentido, o presente artigo tem por objetivo descrever o perfil dos casos de acidentes por animais peçonhentos ocorridos no Brasil, de 2010 a 2019, para verificar a relação entre os biomas brasileiros sobre o número de notificações.

\section{MÉTODO}

Trata-se de um estudo exploratório, baseado em dados secundários coletados do Departamento de Informática do Sistema Único de Saúde (DATASUS) ${ }^{7}$, sobre acidentes causados por animais peçonhentos ocorridos nos Estados e Distrito Federal do Brasil, durante o período de 2010 a 2019. As informações selecionadas foram número absoluto de casos, grupo animal causador do acidente e óbitos. Foi calculada a taxa de letalidade de acidentes por Estado, definida como o número de óbitos notificados/número de casos notificados $\times 100$. 
Foi construído mapa coroplético com a distribuição de número de casos absolutos por Estado e Distrito Federal, considerando os valores acumulados ocorridos durante 0 período de observação e superposição dos limites dos seis biomas brasileiros ${ }^{6}$.

Para estimar a relação entre o desmatamento e o número de casos, foi aplicada a correlação linear de Pearson, utilizando dados do número de casos absolutos por Estado ao ano (valor de $\mathrm{x}$ ) e dados de perda de cobertura arbórea por Estado ao ano (valor de y). Foi considerada correlação positiva quando o valor de $r^{2}$ encontrado foi acima de 1.0, ausente quando $r^{2}$ foi igual a zero $e$ negativa quando $r^{2}$ foi menor que 1.0. Para categorizar a força da correlação, foi estabelecida como correlação forte qualquer valor de $r^{2} \geq 0.7$ para correlações positivas e $\leq-0.7$ para correlações negativas.

Toda a inferência estatística foi realizada no software GraphPad Prism $6.0^{T M}$. Os mapas foram gerados no software Qgis 2.18.20, com posterior edição no software CorelDraw 2019. Para a correlação linear de Pearson foi considerado $p$-valor significativo $\leq 0.05$.

Devido à pesquisa utilizar dados obtidos por fontes secundárias, disponibilizados livremente para o público, não foi necessária submissão e aceite por Comitê de Ética em Pesquisa, de acordo com as atuais resoluções do Conselho Nacional de Saúde ${ }^{8}$.

\section{RESULTADOS}

De acordo com as notificações registradas no DATASUS 7 , entre 2010 e 2019 ocorreram 1.833 .482 casos novos de acidentes por animais peçonhentos no Brasil. Os dois Estados com maior número de casos no país, Minas Gerais e São Paulo, estão distribuídos entre os biomas mata atlântica e cerrado, com números de notificações superiores a 264 mil. Em seguida, em número de notificações, destaca-se Pernambuco (BR), Bahia (BR) e Santa Catarina (BR), inseridos total ou parcialmente nos biomas mata atlântica e caatinga (Figura 1).

No período, houve maior expressão dos registros de acidentes envolvendo escorpiões (52,14\%), majoritariamente concentrados entre Minas Gerais (BR), São Paulo (BR), Bahia (BR) e Pernambuco (BR), que se inserem na transição entre mata atlântica, cerrado e caatinga. Nos acidentes por aranha $(16,23 \%)$, em que houve maior percentual acumulado entre o Paraná (BR), Santa Catarina (BR) e Rio Grande 
do Sul (BR), sendo os dois primeiros totalmente inseridos no bioma mata atlântica e o terceiro em transição entre este e o pampa, houve destaque para o gênero Loxosceles, que ultrapassou $60 \%$ das causas de notificações. Nos acidentes envolvendo serpentes
$(15,45 \%)$, com importante percentual acumulado no bioma amazônia, principalmente no Amapá (BR), Amazonas (BR), Pará (BR) e Roraima (BR), destaca-se o gênero Bothrops como agente causador de $66,14 \%$ dos acidentes (Figuras 2 e 3 ).
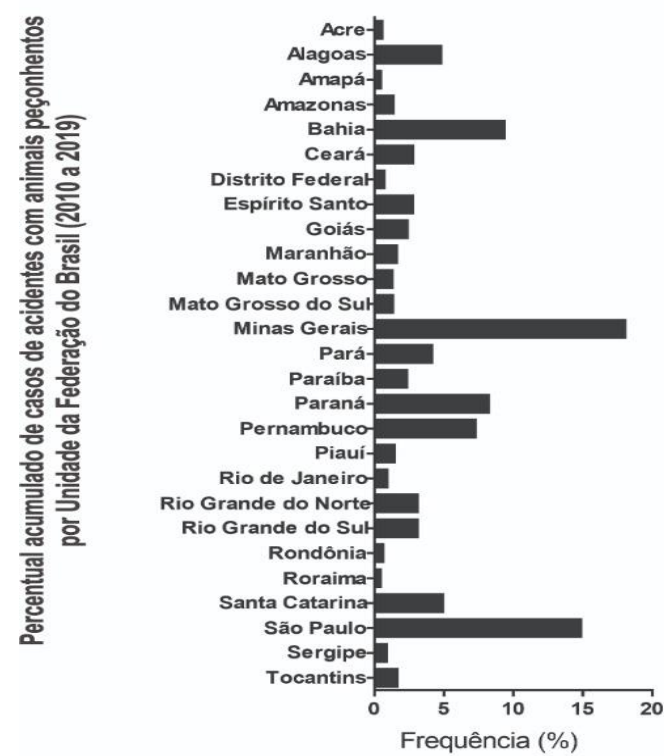

Número acumulado de casos de acidentes com animais peçonhentos por Unidade da Federação do Brasil (2010 a 2019)

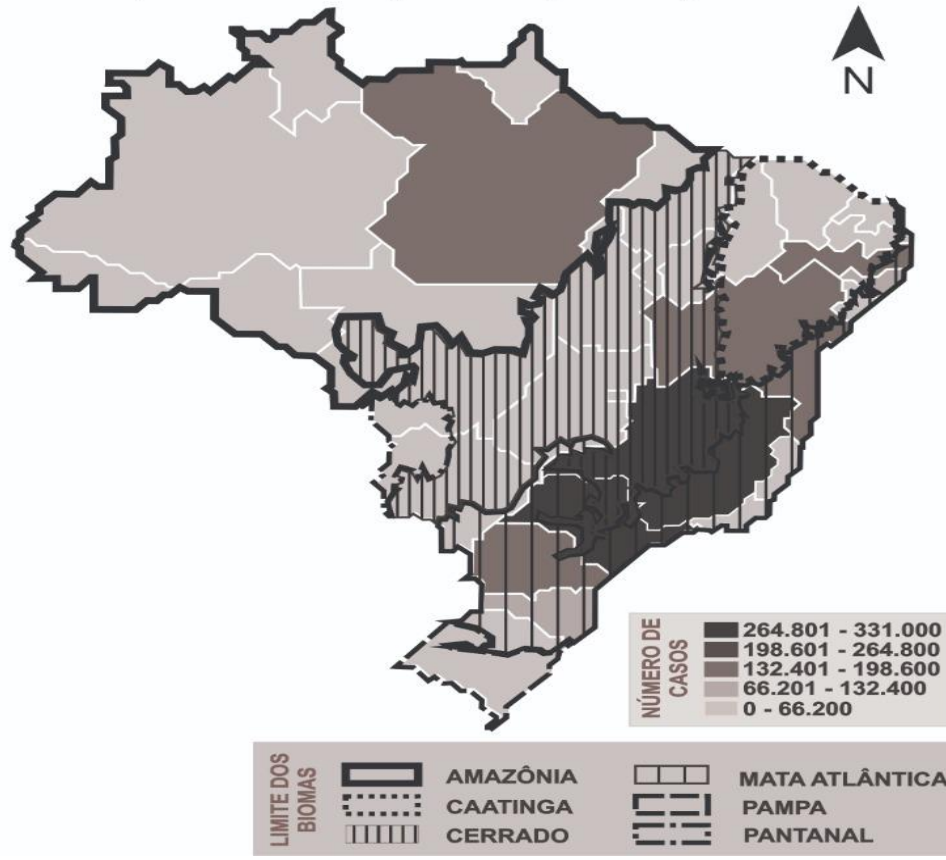

Figura 1 - Número acumulado de casos de acidentes com animais peçonhentos por Unidade da Federação do Brasil, de 2010 a 2019. Fonte: Dados epidemiológicos obtidos do DATASUS. Brasil, 2020.

Os Estados que apresentaram o maior número de casos novos acumuladamente foram Minas Gerais (49.716), São Paulo (41.428), Bahia (24.617) e Pernambuco (21.144).

Aqueles que tiveram maior taxa de letalidade associada aos acidentes, compreendida entre $1 \%$ e maior que $2 \%$, tiveram acidentes mais expressivamente causados por serpentes. Foram estes,
Amapá (BR), Mato Grosso (BR) e Roraima $(B R)$, seguido por São Paulo (BR), onde predominou o escorpionismo. Logo, a maior letalidade está compreendida integral ou parcialmente nos biomas amazônia, cerrado e mata atlântica (Figura 4). 
Principais animais peçonhentos causadores de acidentes notificados no Brasil (2010 a 2019)

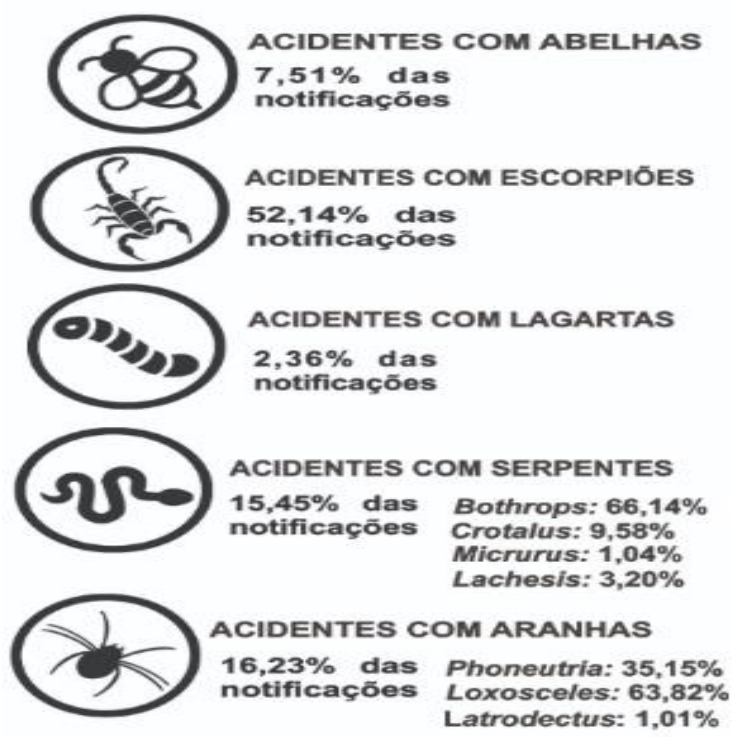

Figura 2 - Representatividade dos acidentes por animal peçonhento no Brasil de 2010 a 2019. Fonte: Dados epidemiológicos retirados do DATASUS. Brasil, 2020.

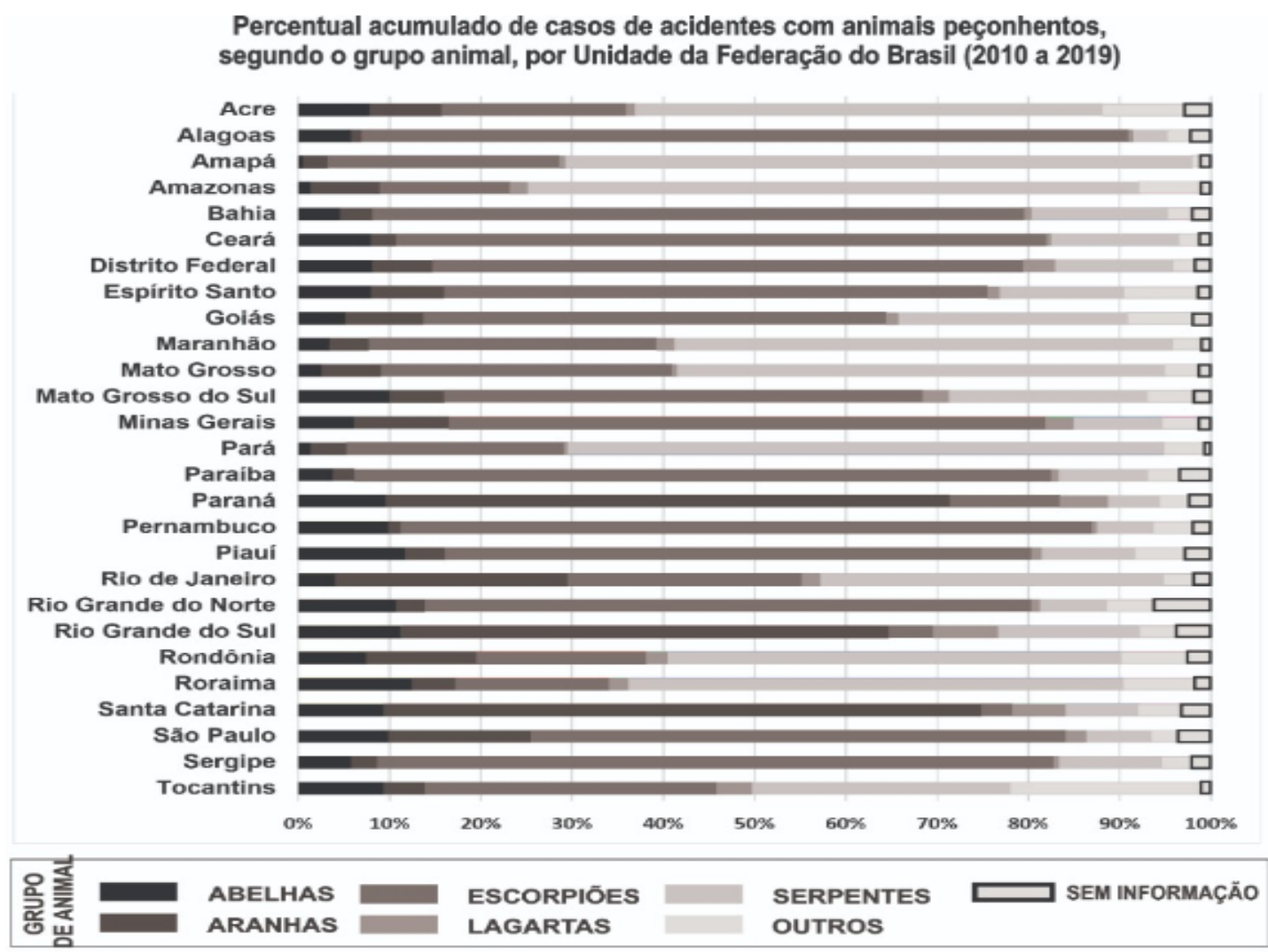

Figura 3 - Distribuição dos animais causadores de acidentes por Estado acumulados e anuais no Brasil de 2010 a 2019. Fonte: Dados epidemiológicos obtidos do DATASUS. Brasil, 2020. 


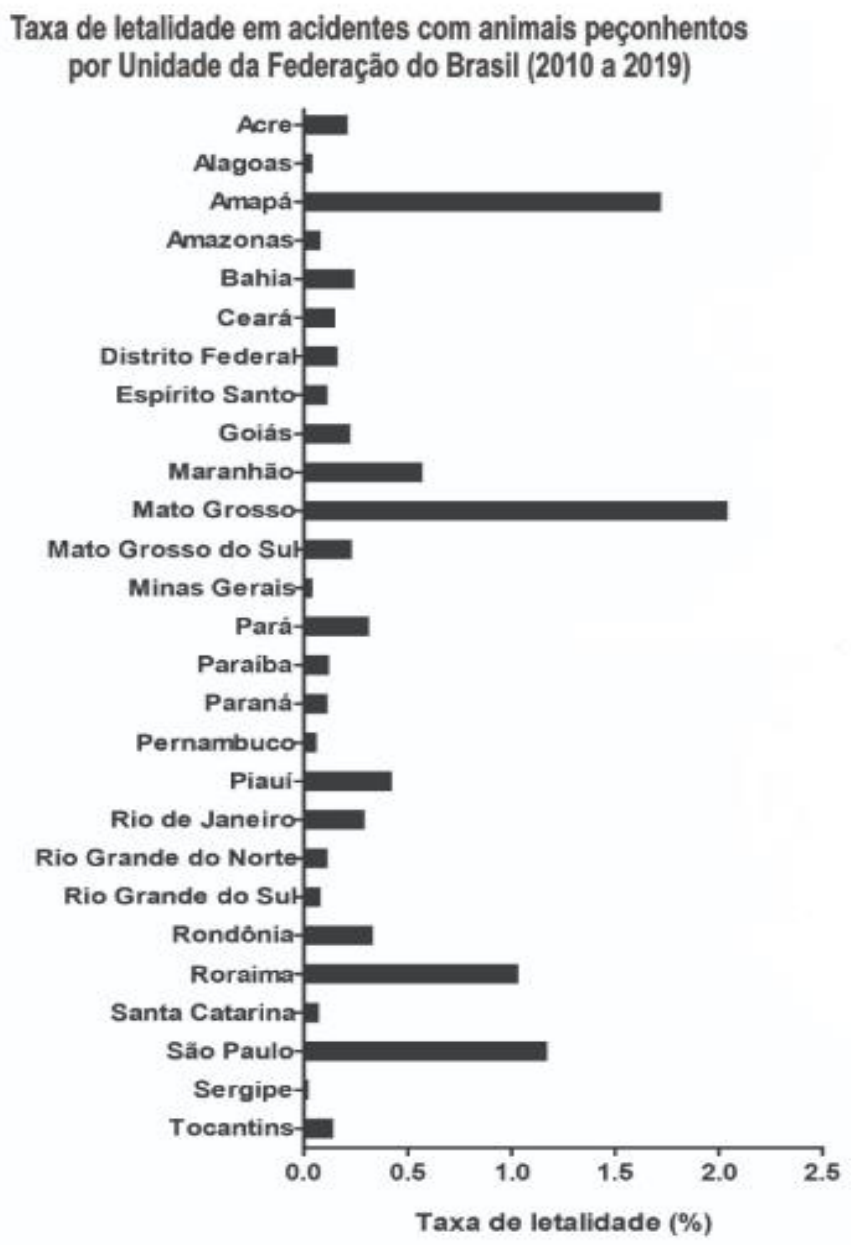

Figura 4 - Taxa de letalidade dos acidentes por animais peçonhentos no Brasil de 2010 a 2019. Fonte: Dados epidemiológicos obtidos do DATASUS. Brasil, 2020.

Ao avaliar a correlação entre desmatamento com base na perda de hectares de cobertura arbórea, e número de casos, foi verificado que muitos Estados mostraram correlação negativa, onde as variáveis se apresentaram como grandezas inversamente

proporcionais, principalmente para os Estados que tiveram mais casos notificados ( $p$-valor $<0.05$ para Bahia e Rio Grande do Norte). Contudo, houve correlação forte negativa, apenas no estado da Bahia (Tabela 1). 
Tabela 1 - Correlação entre desmatamento (através de perda de hectares de cobertura arbórea) e número de notificações de acidentes por animais peçonhentos por Estado no Brasil, de 2010 a 2019.

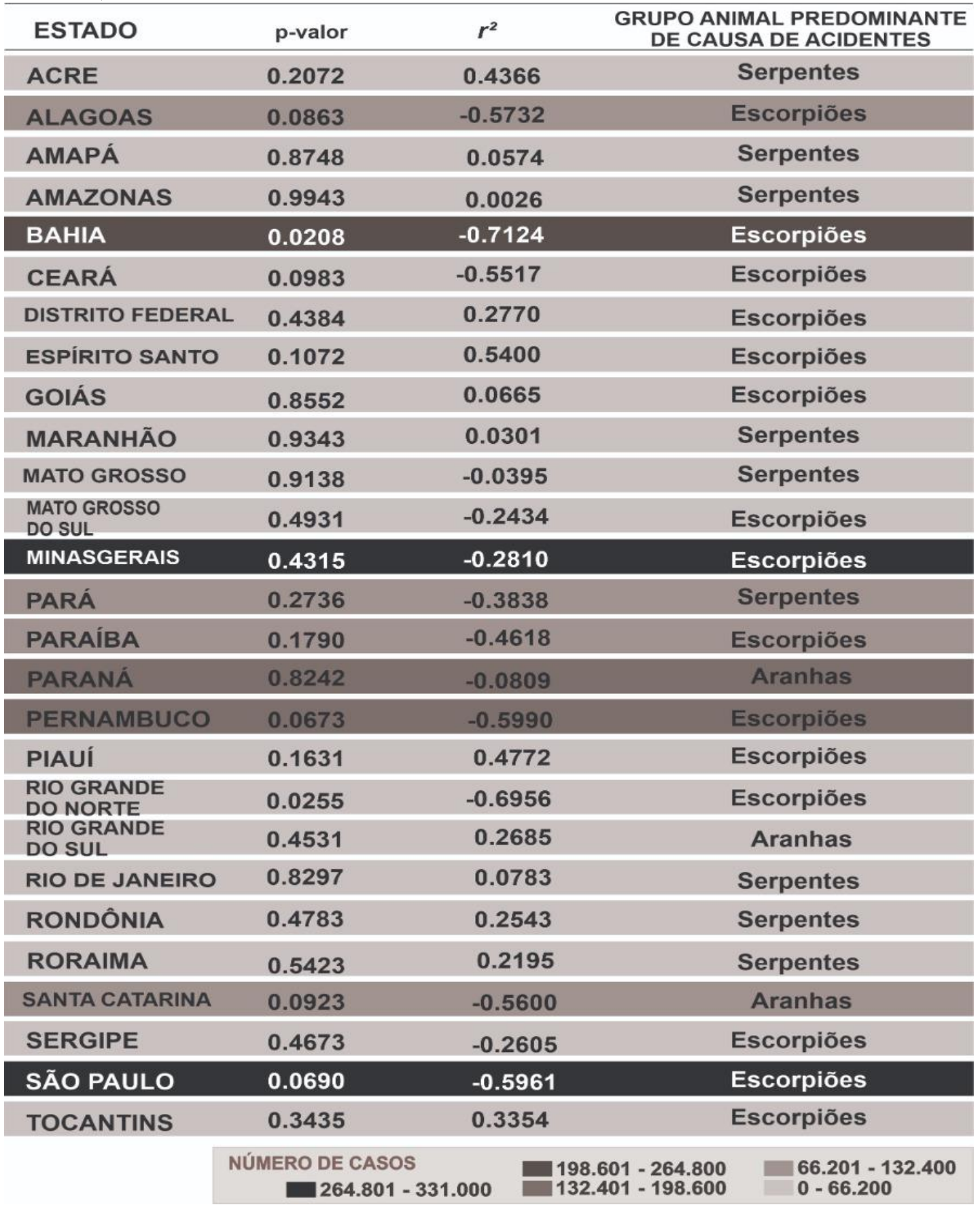

Fonte: Dados epidemiológicos obtidos do DATASUS. Brasil, 2020.

\section{DISCUSSÃO}

As notificações expressas de 2010 a 2019 pelo presente estudo demonstraram casos distribuídos por todos os Estados e Distrito Federal, de forma heterogênea. Apesar de o bioma amazônia apresentar a maior extensão dentre todos, os biomas com número mais expressivo de casos foram cerrado 
e mata atlântica, que tampouco apresentaram maior letalidade associada aos casos, tendo como animal peçonhento predominante o escorpião. A perda de hectares de cobertura arbórea sobre o número de casos teve correlação negativa em grande parte dos Estados, considerada forte apenas na Bahia (BR), que é um dos Estados com maior número de notificações no período.

No estudo, o bioma Amazônia, que comporta a maior floresta tropical do mundo e abrange cerca de $50 \%$ do território nacional, conteve o maior número de acidentes ofídicos, consoante a outros estudos, em que este tipo de acidente predominou9,10. A riqueza de fauna e flora existente na amazônia movimenta uma economia que aproxima o homem do habitat de serpentes, como a pesca e, principalmente, o extrativismo não madeireiro ${ }^{11}$.

Nesse sentido, acidentes por serpentes ocorridos nesse território, normalmente em zonas rurais, quando associados à demora na chegada de atendimento médico adequado podem ser potencialmente danosos, especialmente para crianças, já tendo sido descrito acidente vascular hemorrágico em paciente pediátrico após picada de serpente na árvore nativa Euterpe oleracea durante coleta do fruto açaí ${ }^{12,13}$. Ainda assim, a maior parte dos Estados que compõem este bioma apresentou baixo número de casos de acidentes por animais peçonhentos em relação às demais Unidades Federativas.

Sobre os acidentes ofídicos, dentre as espécies existentes no Brasil somente as famílias Elapidae e Viperidae são peçonhentas ${ }^{14,15}$. Assim como registrado no Nordeste do Brasil ${ }^{16}$, a espécie venenosa Bathrops predominou no presente estudo. No Norte do país, principalmente no bioma Amazônia, há predomínio de elevada pluviosidade nos meses de novembro a março. Nesses períodos, a relação entre temperatura, pluviosidade e a maior exposição das serpentes pode explicar a maior ocorrência dos casos em localidades nas quais há mais atividades em ambientes que podem favorecer o contato com esses animais, devido à sua capacidade de mobilidade consideravelmente superior à dos demais grupos animados aqui destacados ${ }^{16,17}$.

O cerrado, conhecido por ser a savana mais rica em biodiversidade de fauna e flora do mundo, é o segundo maior bioma do Brasil, com cerca de $21 \%$ do território nacional ${ }^{9}$. Tal bioma abrange parte dos três Estados que apresentam o maior número de casos acumulados de acidentes por animais 
peçonhentos, bem como os maiores percentuais dos casos totais para os referidos anos: Minas Gerais (BR), São Paulo (BR) e Bahia (BR). Em boletim epidemiológico do Ministério da Saúde, significativa quantidade de casos de acidentes de trabalhadores do campo, floresta e águas por animais peçonhentos foi registrada nesses três Estados entre 2007 e 2017, representando a maioria dos casos por região em que cada um deles se localiza $^{18}$. 0 predomínio de acidentes causados por escorpiões nesse bioma condiz como habitat predominante para este grupo animal e seu predomínio nessas regiões é corroborado por outros estudos ${ }^{19,20}$.

A mata atlântica é o bioma predominante no litoral, ocupando 15\% do território brasileiro, e é o mais ameaçado, uma vez que sua distribuição atravessa os principais centros urbanos de ocupação humana, já consolidados desde o período de colonização do país ${ }^{9}$. Este fator é expresso pela baixa frequência de notificações no Espírito Santo (BR) e Rio de Janeiro (BR), que são áreas costeiras já consolidadas como conglomerados de predominância urbana. Desta forma, os números de casos apresentados nessas localidades relacionados a este bioma podem estar atrelados a áreas de interior e, principalmente, em transição com os biomas vizinhos. Desta forma, a mata atlântica engloba Estados que, mesmo de forma descontínua, formam um corredor que abarca o maior número de casos no país, mas que tenderiam a ser importados de sua área de transição com os demais biomas.

A caatinga, bioma que se encontra majoritariamente no Nordeste brasileiro, engloba aproximadamente $11 \%$ de todo o território nacional?. Excetuando-se Bahia (BR) e Pernambuco (BR), que apresentam territórios pertencentes à mata atlântica, os demais Estados possuem ambiente propício à disseminação de espécies de escorpiões, condizendo com os dados apresentados neste trabalho ${ }^{19}$.

Dentre os escorpiões presentes no Brasil, os pertencentes ao gênero Tityus são perigosos e causadores de acidentes. Sobre os escorpiões de interesse médico no território nacional, são levantados fatores que podem contribuir para sua distribuição no território. Dentre eles, cita-se a possibilidade de o padrão de distribuição da população original em determinadas localidades ter ampliado por ações antrópicas das quais decorreu uma seleção negativa de espécies 
especialistas, em detrimento de espécies oportunistas $^{21}$.

Referente ao bioma pampa, que compreende a região do extremo Sul do país, estes apresentaram acidentes causados por aranhas como maioria, achado compatível a outras literaturas ${ }^{22,23}$. Dentre os três gêneros de aranhas destacados no presente estudo, as do Loxoceles, mais expressivo, são pouco agressivas, havendo a picada somente com a sua compressão ao corpo. Além disso, Latrodectus têm hábitos domiciliares, embora também possam residir em arbustos e gramíneas ${ }^{6}$.

Embora acidentes com abelhas e lagartas tenham apresentado notificação em todos os biomas do país, estes apareceram com os menores índices. Os dados referentes às abelhas contrastam com o estudo por Terças et $\mathrm{al}^{24}$, que revela aumento anual dos casos de acidentes por abelhas africanas no país, chegando ao pico de 12.660 casos em 2014, com tratamento de casos graves dependente apenas de suporte clínico ${ }^{24}$.

O maior quantidade de acidentes por lagartas ocorreu em Santa Catarina (bioma mata atlântica) e Rio Grande do Sul (entre biomas mata atlântica e pampa). Nesse sentido, essa classe de acidentes teve menor relevância, embora alguns casos tenham sido

registrados em Minas Gerais ${ }^{25}$. Esses acidentes apresentam menor letalidade, seja pela subnotificação como pelo bom prognóstico em relação aos demais acidentes $^{6}$.

Embora Machado 4 levante na sua análise sobre um panorama relativo aos acidentes por animais peçonhentos para o Brasil o desmatamento como um fator preponderante para o aumento de casos de acidentes no país, devido aos animais serem vítimas de um deliberado processo de desequilíbrio ecológico, dados do Global Forest Whatch ${ }^{26}$ revelaram para o período de 2010 a 2019 que muitas regiões apresentaram correlação negativa, e somente a Bahia (BR) apresentou correlação forte. Nesse sentido, não é possível afirmar que a perda de cobertura vegetal possa ocasionar a migração das espécies animais e possibilidade maior de ocorrência de acidentes.

As limitações do presente estudo são inerentes à fonte dos dados, que depende do correto preenchimento pelos profissionais de saúde, bem como pode estar subestimada em relação à realidade nacional.

\section{CONCLUSÃO}


Os biomas que apontaram maior número de acidentes por animais peçonhentos entre 2010 e 2019 no Brasil foram mata atlântica e cerrado, nos quais os Estados com maior notificação se situam, parcial ou integralmente, em zona de transição com o bioma caatinga. Acidentes envolvendo escorpiões foram os que apresentaram a maior prevalência para o período, na maioria dos biomas brasileiros, com enfoque no cerrado e na caatinga, podendo significar um potencial problema de saúde pública devido sua fácil adaptação ao ambiente urbano e aparente independência de cobertura arbórea para dispersão. As aranhas foram as responsáveis pelo segundo maior número de registros, com prevalência maior para os biomas mata atlântica e pampa, seguidas pelos casos de acidentes ofídicos, que abarcaram a maior taxa de letalidade no período estudado. Acidentes com abelhas e lagartas mostram os menores índices de notificações, sem expressividade quanto ao número de casos registrado, para os anos observados. Ademais, os dados apresentados não demonstram correlação positiva entre desmatamento e aumento de notificações de acidentes por animais peçonhentos.

Ressalta-se neste trabalho a elevada notificação de acidentes causados por escorpiões, tanto por Estado quanto o acumulado de casos novos ao final do período de observação. Frente a esses achados, há possibilidade de se determinar pontos estratégicos para vigilância epidemiológica dos acidentes por animais peçonhentos. Além disso, visando mitigar subnotificação dos casos, deve ser estimulado o registro completo dos casos diagnosticados no dia a dia da prática assistencial pela equipe de saúde, oportunizando a estruturação de unidades de atendimento aos acidentados, principalmente nas localidades mais inóspitas e até então com menos notificações, enfatizadas no presente estudo.

\section{REFERÊNCIAS}

1. Ministério do Meio Ambiente (BR). Secretaria de Biodiversidade e Florestas. Fragmentações de Ecossistemas: Causas, Efeitos sobre a Biodiversidade e Recomendações de Políticas Públicas. Brasília: Ministério do Meio Ambiente; 2003.

2. Barraviera B. O ensino dos acidentes por animais peçonhentos nas escolas médicas brasileiras. Rev Soc Bras Med Trop. 1992; 25(3):203-204. 
3. Brasil. Manual de Primeiros Socorros. Fundação Oswaldo Cruz. Núcleo de Biossegurança. Rio de Janeiro: FIOCRUZ; 2003.

4. Machado C. Um panorama dos acidentes por animais peçonhentos no Brasil. J Health NPEPS. 2016; $1(1): 1-3$.

5. IBGE. Instituto Brasileiro de Geografia e Estatística. Biomas. 2019. Disponível em: https://www.ibge.gov.br/geoci encias/informacoes-

ambientais/15842-

biomas.html?=\&t=downloads.

6. Ministério da Saúde (BR). Manual de Diagnóstico e Tratamento de Acidentes por Animais Peçonhentos. Acessoria de Comunicação e Educação em Saúde. Brasília: Ministério da Saúde; 2001.

7. Ministério da Saúde (BR). DATASUS Informações de Saúde, Epidemiológicas e Morbidade: banco de dados. Brasília: Ministério da Saúde; 2020 [acesso em 2020 Dez 22]. Disponível em:

http://tabnet.datasus.gov.br/cgi/defto htm.exe?sinannet/cnv/animaispa.def.

8. Brasil. Conselho Nacional de Saúde. Resolução $n^{\circ} 580$, de 22 de março de 2018. Regulamenta o disposto no item XIII.4 da Resolução CNS n 466 , de 12 de dezembro de 2012, que estabelece que as especificidades éticas das pesquisas de interesse estratégico para o Sistema Único de Saúde (SUS) serão contempladas em Resolução específica, e dá outras providências. Diário Oficial da União, Brasília, 16 jul. 2018. Seção 1, p.55.

9. Ministério do Meio Ambiente (BR). Secretaria Nacional de Biodiversidade e Florestas. Áreas Prioritárias para Conservação, Uso Sustentável e Repartição de Benefícios da Biodiversidade Brasileira, Atualização: Portaria MMA $\mathrm{n}^{\circ}$ 9, de 23 de janeiro de 2007. Brasília: Ministério do Meio Ambiente; 2007.

10. Matos RR, Ignotti $E$. Incidência de acidentes ofídicos por gêneros de serpentes nos biomas brasileiros. Ciênc saúde coletiva. 2020; 25(7):2837-2846.

11. Castro E. Dinâmica socioeconômica e desmatamento na Amazônia. Novos Cad NAEA. 2005; 8(2):5-39.

12. Silva AM, Sachett J, Monteiro WM, Bernarde PS. Extractivism of palm tree fruits: A risky activity because of snakebites in the state of Acre, Western Brazilian Amazon. Rev Soc Bras Med Trop. 2019; 52:1-4.

13. Pardal PPO, Pinheiro ACJS, Silva CTC, Santos PRSG, Gadelha MAC. 
Hemorrhagic stroke in children caused by Bothrops marajoensis envenoming: A case report. J Venom Anim Toxins Incl Trop Dis. 2015; 21:53.

14. Nogueira CC, Alencar JPSV, Argolo AJS, Arredondo JC, Arzamendia V, Azevedo JA, et al. Atlas of Brazilian Snakes: verified point locality maps for mitigating the Wallacean shortfall in a megadiverse snake fauna. South Am J Herpetol. 2019; 14(1):1-274.

15. Melgarejo AR. Serpentes Peçonhentas do Brasil. In: Cardoso JLC, França OSF, Wen FH, Málaque CMS, Haddad Júnior V. Animais peçonhentos no Brasil: Biologia, clínica e terapêutica dos acidentes. São Paulo: Sarvier; 2009.

16. Costa MKB, Fonseca CS, Navoni JA, Freire EMX. Snakebite accidents in Rio Grande do Norte state, Brazil: Epidemiology, health management and influence of the environmental scenario. Trop Med Int Heal. 2019; 24(4):432-441.

17. Oliveira RC, Wen FH, Sifuentes DN. Epidemiologia dos acidentes por animais peçonhentos. In: Cardoso JL, Haddad-Jr V, França FOS, Wen FH, Malaque CMS. Animais peçonhentos do Brasil: biologia, clínica e terapêutica. São Paulo: Sarvier; 2009.

18. Ministério da Saúde (BR). Secretaria de Vigilância em Saúde. Acidentes de trabalho por animais peçonhentos entre trabalhadores do campo, floresta e águas, Brasil 2007 a 2017. Brasília: Ministério da Saúde; 2019.

19. Gonçalves JE, Medeiros SMFRS, Cavalcanti IDL, Mendes RCMG, Bezerra INM, Nóbrega MM, et al. Accidents caused by venomous animals: an analysis of the epidemiological profile in the Northeast region of Brazil in the period from 2010 to 2019. RSD. 2020; 9(10):e4679108843.

20. Silva PLN, Costa AA, Damasceno RF, Oliveira Neta Al, Ferreira IR, Fonseca AD. Perfil epidemiológico dos acidentes por animais peçonhentos notificados no Estado de Minas Gerais durante o período de 20102015. Rev Sustinere. 2017; 5(2):199217.

21. Lourenço WR, Von Eickstedt VRD. Escorpiões de Importância Médica. In: Cardoso JLC, França FOS, Wen FH, Málaque CMS, Haddad JRV. Animais Peçonhentos no Brasil: Biologia, Clínica e Terapêutica dos Acidentes. São Paulo: Sarvier, 2;09. 
22. Schier DT, Lemos MR, Campos CGC, Cardoso JT. Estudo sobre a influência de variáveis metereológicas nos casos de acidentes por animais peçonhentos em Lages - SC. Hygeia. 2019; 15(31):43 -55.

23. Chagas F, D'Agostini F, Beltrame V. Aspectos epidemiológicos dos acidentes por aranhas no Estado do Rio Grande do Sul, Brasil. Evid Biotecnol Aliment. 2010; (1):121-30.

24. Terças ACP, Vivi VK, Machado C, Lemos ERS. Aspectos epidemiológicos dos acidentes por picada de abelha africana. J Health NPEPS. 2017; 2(Supl.1):58-72.

25. Silveira JL, Machado C. Epidemiologia dos acidentes por animais peçonhentos nos municípios do sul de Minas Gerais. J Health NPEPS. 2017; 2(Supl.1):88-101.

26. Global Forest Watch. Banco de Dados [acesso em 2020 Abr 16]. Disponível em: https:/ / www.globalforestwatch.org/ dashboards/country/BRA/?category= summary\&dashboardPrompts=eyJzaG 93UHJvbXB0cyl6dHJ1ZSwicHJvbXB0c 1ZpZXdIZCI6WyJkYXNoYm9hcmRBbm FseXNlcylsImRvd25sb2FkRGFzaGJvYX JkU3RhdHMiLCJzaGFyZVdpZGdldCIsIn dpZGdldFNldHRpbmdzliwidmlld05hd
GlvbmFsRGFzaGJvYXJkcyJdLCJzZXR0 aW5ncyl6eyJvcGVuljpmYWxzZSwic3R lcEluZGV4ljowLCJzdGVwc0tleSI6liJ9f Q\%3D\%3D\&firesAlertsCumulative $=$ ey $J$ pbnRlcmFjdGlvbil6e319\&firesAlertsHi storical=eyJpbnRlcmFjdGlvbil6e319\& firesAlertsStats=eyJpbnRlcmFjdGlvbil 6e319\&gladAlerts=eyJsYXRIc3REYXRII joiMjAxOSOWOCOxMilsImludGVyYWNO aW9uljp7fX0\%3D\&location=WyJjb3Vu dHJ5liwiQIJBII0\%3D\&map=eyJjZW50Z XliOnsibGFOljotMTluMjY5NDkONzkyNT k5NzMyLCJsbmciOi000S440DU5MTY3 NDcxNTg2N30sInpvb20iOjluMjcyNTI4 ODI1OTI1MzkONiwiY2FuQm91bmQiO mZhbHNLLCJkYXRhc2VOcyl6W3siZGF0 YXNIdCI6IjBiMDIwOGI2LWIOMjQtNGI1 Ny05ODRmLWNhZGRmYTI1YmEyMilsI mxheWVycyl6WyJjYzM1NDMyzCOzOG Q3LTRhMDMtODcyZSOzYTCXYTJmNTU 1ZmMiLCJiNDUZNTBIMy01YTC2LTQ0Y 2QtYjBhOS01MDM4YTBkOGJmYWUiXS wiYm91bmRhcnkiOnRydWUsIm9wYW NpdHkiOjEsInZpc2liaWxpdHkiOnRyd WV9LHsiZGFOYXNIdCI6ImI10DQ5NTRj LTBkOGQtNDBjNi04NTljLWYZZmRmM 2MyYzVkZilsImxheWVycyl6Wyl0OWE4 MGU3MC1IYzUyLTRIZjgtYmNjNi1mYjl 3NzFkOTViMmMiXSwib3BhY2lOeSI6MS widmlzaWJpbGl0eSI6dHJ1ZSwicGFyY W1zljp7InRocmVzaCl6MzAsInZpc2lia WxpdHkiOnRydWV9fV19\&path=eylwlj oiZGFzaGJvYXJkcylsljEiOiJjb3VudHJ5 
liwiMil6IkJSQSJ9\&treeLoss=eyJpbnRlc mFjdGlvbil6e319\&treeLossPct=eyJoa WdobGInaHRIZCI6ZmFsc2UsImludGVy
YWNOaW9uljp7fX0\%3D\&treeLossTsc= eyJoaWdobGlnaHRIZCI6ZmFsc2V9.

Financiamento: Os autores declaram que não houve financiamento.

Conflito de interesses: Os autores declaram não haver conflito de interesses.

\section{Participação dos autores:}

- Concepção: Moraes FCA, Silva AR, Silva ER, Coelho JS, Pardal PPO.

- Desenvolvimento: Moraes FCA, Silva AR, Silva ER, Coelho JS, Pardal PPO.

- Redação e revisão: Moraes FCA, Silva AR, Silva ER, Coelho JS, Pardal PPO.

Como citar este artigo: Moraes FCA, Silva AR, Silva ER, Coelho JS, Pardal PPO. Relação dos biomas nos acidentes peçonhentos no Brasil. J Health NPEPS. 2021; 6(1):175-790.

Submissão: $22 / 02 / 2021$

Aceito: $12 / 05 / 2021$

Publicado: 01/06/2021 